\title{
Temperature dependence of second-order nonlinear relaxation of a poled chromophore-doped sol-gel material
}

\author{
Hélène Goudket, ${ }^{\text {a) }}$ Michael Canva, and Yves Lévy \\ Laboratoire Charles Fabry de l'Institut d'Optique Théorique et Appliquée, Centre National de la Recherche \\ Scientifique, CNRS UMR 8501, Centre Universitaire d'Orsay-Paris XI, BP 147, 91403 Orsay, France \\ Frédéric Chaput and Jean-Pierre Boilot \\ Laboratoire de Physique de la Matière Condensée, Centre National de la Recherche Scientifique, \\ CNRS UMR 7643, Ecole Polytechnique, 91128 Palaiseau, France
}

(Received 11 June 2001; accepted for publication 12 September 2001)

\begin{abstract}
Second-order optical nonlinearity relaxations of hybrid inorganic/organic sol-gel films doped with poled Disperse Red 1 chromophores were measured by second harmonic generation at several temperatures. The decay curves were fitted to biexponentials and Köhlrausch, Williams, and Watts (KWW) stretched exponentials. It can be concluded that the KWW model well describes chromophore thermal reorientation in hybrid sol-gel materials, as is known for all-organic polymers. Using the Arrhenius law, the activation energy of the reorientation process has been determined to be about 50 or $70 \mathrm{kcal} \mathrm{mol}^{-1}$. A stability of roughly a decade has been extrapolated at room temperature. Another experiment at room temperature gave an estimation of the stability that was found to be in qualitative agreement. It is shown that poled chromophore-doped sol-gel materials have the potential to be stable enough for applications in telecommunication devices. (C) 2001 American Institute of Physics. [DOI: 10.1063/1.1416865]
\end{abstract}

\section{INTRODUCTION}

Organic and organomineral hybrid polymers are increasingly studied for application in nonlinear optics. Materials possessing a strong $\chi^{(2)}$ nonlinearity are particularly prized for second-order nonlinear applications such as electro-optic modulation, ${ }^{1}$ and active doped polymer components are currently on the verge of being commercial products. To reach this point, a great deal of work has been devoted to increase the microscopic nonlinearity coefficients of chromophores and their large macroscopic orientation in polymeric environment. But the strong $\chi^{(2)}$ that has been obtained must also be maintained in operational conditions for a long period of time: a decade or more. In purely organic materials, several studies have been reported concerning the lifetime of second-order nonlinearity. ${ }^{2,3}$ This article reports on the study of second-order nonlinearity thermal stability in chromophore doped organomineral thin films of sol-gel type, for which fewer communications ${ }^{4-6,22}$ have already been published.

In order to evaluate the lifetime of the second-order nonlinearity in Corona poled sol-gel thin films, we monitored the second harmonic generation (SHG) signal evolution with time. However, as needed for telecommunication requirements, decades would be necessary for experiments at room temperature. To speed up the process, SHG relaxation has been studied at higher temperatures, below the poling temperature where the chromophore orientation is known to occur within a few hours. We confront the recorded experimental data with different exponential-based models whose

\footnotetext{
a) Author to whom correspondence should be addressed; electronic mail: helene.goudket@iota.u-psud.fr
}

accuracy is then compared. The temperature dependence of the time constant allows the determination of the activation energy of the reorientation process. An extrapolation toward room temperature leads to a theoretical value of the actual relaxation time of the sol-gel samples, depending slightly on the model used. At room temperature, an experimental SHG relaxation time recorded over more than a year makes a rough estimation of the nonlinear stability at room temperature possible.

\section{MATERIAL AND EXPERIMENTAL SETUP}

Hybrid films studied during this set of experiments were prepared according to a previously published procedure. ${ }^{7}$ Organosilicon polymers containing nonlinear (Disperse Red 1: DR1) and carbazole pendant groups were synthesized from functionalized monomers copolymerized with tetraethoxysilane (TEOS) through sol-gel reactions. Samples were obtained with the following molar ratios: 5 carbazole/1 DR1/1 TEOS. After deposition by a conventional spin-coating technique, the films were heat treated at $120{ }^{\circ} \mathrm{C}$ for several hours.

The variations of second-order nonlinearity were observed through second harmonic generation (SHG) generated in the thin film by a $1.06 \mu \mathrm{m}$ nanosecond-pulsed $\mathrm{Nd}$ :yttrium-aluminum-garnet beam incident at about $50^{\circ}$. All measurements were referenced to a quartz crystal's SHG. Temperature and SHG were controlled and recorded through a computer program.

The samples were previously Corona poled at $90{ }^{\circ} \mathrm{C}$ : the sample was brought to poling temperature $\left(T_{p}=90^{\circ} \mathrm{C}\right)$ and left to thermalize $(20 \mathrm{~min})$, then the Corona discharge of about $3.5 \mathrm{kV}$ was activated. When the SHG reached a plateau (20-30 min later), the sample was left to cool with the 


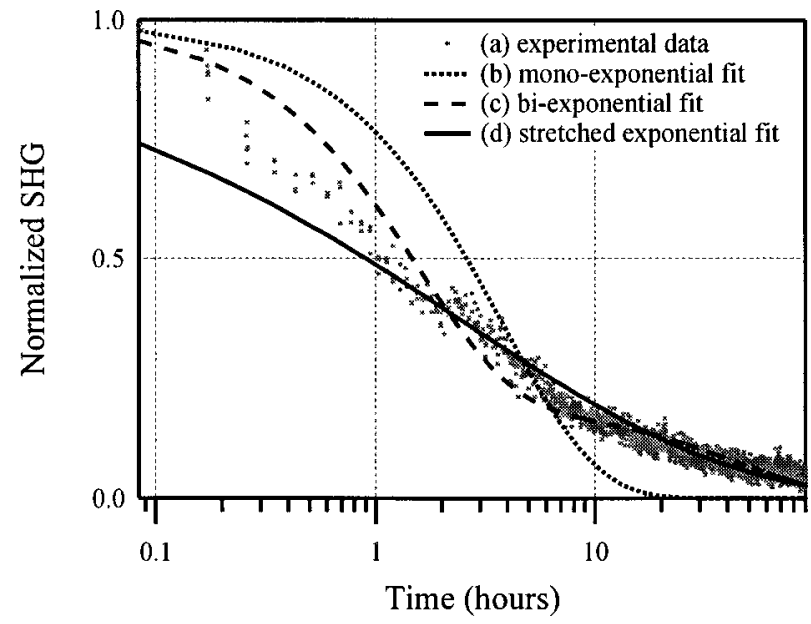

FIG. 1. (a) Experimental data obtained at $72{ }^{\circ} \mathrm{C}$ plotted on a logarithmic time scale. (b) Monoexponential fit $\phi_{1}(t)=e^{-t / \tau}$ with $\tau=3.8 \mathrm{~h}$. (c) Biexponential fit $\phi_{2}(t)=K e^{-t / \tau_{1}}+(1-K) e^{-t / \tau_{2}}$ with $\mathrm{K}=0.80 ; \tau_{1}=92 \mathrm{~min} ; \tau_{2}$ $=45 \mathrm{~h}$. (d) Stretched exponential fit $\phi_{3}(t)=e^{-(t / \tau)^{\beta}}$ with $\tau=2.5 \mathrm{~h} ; \beta$ $=0.36$.

Corona field still applied. When the sample reached room temperature, the Corona field was turned off. The sample was then stored for a few weeks maximum in preparation for relaxation measurements. A typical $d_{33}$ nonlinear coefficient of about $100 \mathrm{pm} / \mathrm{V}$ at $1.06 \mu \mathrm{m}$ (still resonant) has been reported. ${ }^{8}$

The SHG decay curves were measured using the same setup. The poled sample was heated up quickly to the measurement temperature, here taken between 63 and $82^{\circ} \mathrm{C}$, and the SHG signal was monitored until almost complete loss. When the relaxation was very slow, the samples were placed in an oven at the given temperature and taken out periodically for later measurements.

\section{EXPERIMENTAL RESULTS AND ANALYSIS}

A typical decay curve obtained during this series of experiments is shown in Fig. 1(a). The relaxation curves have all been analyzed by several standard distributions, which are subsequently compared.

The simplest distribution is the monoexponential expressed as follows:

$$
\phi_{1}(t)=e^{-t / \tau},
$$

where $\tau$ is the relaxation time. This function could have been immediately eliminated, as it describes a material in which every chromophore is in an identical environment. This is not the case in an amorphous sol-gel matrix, as can be seen in Fig. 1(b).

The biexponential distribution is written as

$$
\phi_{2}(t)=K e^{-t / \tau_{1}}+(1-K) e^{-t / \tau_{2}},
$$

where $K$ is the fraction of population reacting with a short relaxation time $\tau_{1}$, and $(1-K)$ the complementary fraction reacting with a longer relaxation time $\tau_{2}$. The sol-gel matrix is therefore assumed to possess two different chromophore environments, which is a very crude approximation in a (hybrid) polymer structure. As suggested by Singer, the experi-
TABLE I. Values of the three fitting parameters obtained with the biexponential distribution $\phi_{2}(t)=K e^{-t / \tau_{1}}+(1-K) e^{-t / \tau_{2}}$ applied to experimental data obtained at temperatures below poling temperature $\left(T_{p}=90^{\circ} \mathrm{C}\right)$.

\begin{tabular}{cccc}
\hline \hline $\begin{array}{c}\text { Temperature } \\
\left({ }^{\circ} \mathrm{C}\right)\end{array}$ & $K$ & $\tau_{1}$ & $\tau_{2}$ \\
\hline 82 & 0.55 & $3 \mathrm{~min}$ & $80 \mathrm{~min}$ \\
81 & 0.83 & $18 \mathrm{~min}$ & $4.3 \mathrm{~h}$ \\
78 & 0.83 & $23 \mathrm{~min}$ & $4 \mathrm{~h}$ \\
76 & 0.72 & $25 \mathrm{~min}$ & $5 \mathrm{~h}$ \\
75 & 0.66 & $32 \mathrm{~min}$ & $6 \mathrm{~h}$ \\
72 & 0.80 & $92 \mathrm{~min}$ & $45 \mathrm{~h}$ \\
68 & 0.73 & $87 \mathrm{~min}$ & $52 \mathrm{~h}$ \\
63 & 0.72 & $6 \mathrm{~h}$ & $430 \mathrm{~h}$ \\
\hline \hline
\end{tabular}

mental decay and its biexponential fit are depicted on a logarithmic time scale in Fig. $1(\mathrm{c}){ }^{3}$ The fitting parameters $K, \tau_{1}$, and $\tau_{2}$ of the biexponential model are reported in Table I. The fit does not follow the same curves as the experimental data. To better fit the experimental data, the addition of new parameters can be envisaged in the form of a multiple exponential, but such a function would be of little interest due to the numerous combinations of parameters that could be chosen.

The standard distribution usually applied to second-order nonlinearity relaxation in polymers is the stretched exponential or Köhlrausch, Williams and Watts (KWW) distribution $^{9-12}$ given by

$$
\phi_{3}(t)=e^{-(t / \tau)^{\beta}},
$$

where $\tau$ is the relaxation time and $\beta$ the dispersion parameter $(0<\beta \leqslant 1)$. (When $\beta$ diminishes from 1 toward 0 , the dispersion of the system increases.)

It is usual to define an average relaxation time as

$$
\langle\tau\rangle=\int_{0}^{\infty} e^{(-t / \tau)^{\beta}} \cdot d t=\frac{\tau \Gamma(1 / \beta)}{\beta},
$$

where $\Gamma$ is the "gamma" function. Applied to experimental data, the KWW distribution fits more closely in logarithmic time scale [Fig. 1(d)], except for very short time values. The values of the two fitting parameters $\tau$ and $\beta$ of the KWW distribution, as well as the average relaxation times $\langle\tau\rangle$ calculated by means of equation [Eq. (4)], can be found in Table II.

TABLE II. Values of the two fitting parameters and average relaxation time obtained with the KWW distribution $\phi_{3}(t)=e^{-(t / \tau)^{\beta}}$ applied to experimental data obtained at temperatures below poling temperature $\left(T_{p}=90^{\circ} \mathrm{C}\right)$.

\begin{tabular}{cccc}
\hline \hline $\begin{array}{c}\text { Temperature } \\
\left({ }^{\circ} \mathrm{C}\right)\end{array}$ & $\tau$ & $\beta$ & $\langle\tau\rangle$ \\
\hline 82 & $20 \mathrm{~min}$ & 0.40 & $66 \mathrm{~min}$ \\
81 & $27 \mathrm{~min}$ & 0.53 & $49 \mathrm{~min}$ \\
78 & $34 \mathrm{~min}$ & 0.61 & $50 \mathrm{~min}$ \\
76 & $50 \mathrm{~min}$ & 0.50 & $1.6 \mathrm{~h}$ \\
75 & $79 \mathrm{~min}$ & 0.51 & $2.6 \mathrm{~h}$ \\
72 & $2.5 \mathrm{~h}$ & 0.36 & $12 \mathrm{~h}$ \\
68 & $3.7 \mathrm{~h}$ & 0.34 & $21 \mathrm{~h}$ \\
63 & $19 \mathrm{~h}$ & 0.29 & $215 \mathrm{~h}$ \\
\hline \hline
\end{tabular}


It must be noted that the results obtained with both the biexponential and KWW models depend on the duration of the measurement. In the case of the biexponential distribution, the longer the measurement is, the higher the time constants are and the lower the proportion $(1-K)$ of chromophores reacting at the long relaxation time $\tau_{2}$ is. In the case of the KWW distribution, the relaxation time $\tau$ does not vary, but the stretching parameter $\beta$ increases when data length decreases. Thus, the average relaxation time also increases with the data length decrease. These remarks are unsurprising, since at short observation times, only the quickest chromophores have relaxed. Thus, the fraction $K$ of the quicker reacting population, compared to the slow reacting population $(1-K)$, will increase all the more as the data length increases. Similarly, the dispersion of the relaxation response remains small ( $\beta$ close to 1$)$ if the measurement is short, and it will increase ( $\beta$ decreases) along with the length of the measurement. In our case, typical data length is around 50 times $\tau$ or 10 times $\langle\tau\rangle(\beta$ varies between 0.3 and $0.5)$.

\section{DISCUSSION}

\section{A. Comparison of the distributions}

As seen above, the monoexponential distribution does not represent well second-order nonlinearity decay curves in sol-gel thin films. Yet, it has sometimes been used to approximate second-order relaxation in poled polymers. ${ }^{13}$

The biexponential model has also been used to describe second-order relaxation, either in polymers, or in sol-gel materials. ${ }^{6}$ An empirical variant of this model has also recently been proposed to describe relaxation behavior. ${ }^{14}$

However, it is quite clear that the KWW distribution has fewer parameters, and is more adequate to account for isothermal chromophore relaxation in our sol-gel thin films. Most dopant orientation relaxation analyses in polymers stand on the KWW distribution.

\section{B. Activation energy of the process}

In Fig. 2, we can observe that the temperature dependence of $\langle\tau\rangle$ (and $\tau$ ) for temperatures below poling temperature, is described by an Arrhenius law

$$
\langle\tau\rangle \propto e^{-E_{a} / k T},
$$

where $k$ is Boltzmann's constant. This is usual for relaxation times obtained at temperatures below glass transition temperature $T_{g}$ in polymers, ${ }^{13,15-19}$ although there are some exceptions. $^{20}$ The activation energy for our side-chain solgel materials is calculated: $E_{a}=71 \mathrm{kcal} \mathrm{mol}^{-1}$ for $\langle\tau\rangle$. This value is well within the range given for doped all-organic polymers $\left(40-80 \mathrm{kcal} \mathrm{mol}^{-1}\right)$ for which the lowest values correspond to guest-host systems and the largest to sidechain polymers, with the noticeable exception of the recent report from Michelotti et al., where $E_{a}=140 \mathrm{kcal} \mathrm{mol}^{-1}$ for an apparently classical DR1 side-chain poly(methyl methacrylate) of $T_{g}=110^{\circ} \mathrm{C}$. ${ }^{19}$ Such high activation energies are expected to be found for cross-linked polyimide materials of high $T_{g}$. It should be noted that the activation energy calcu-

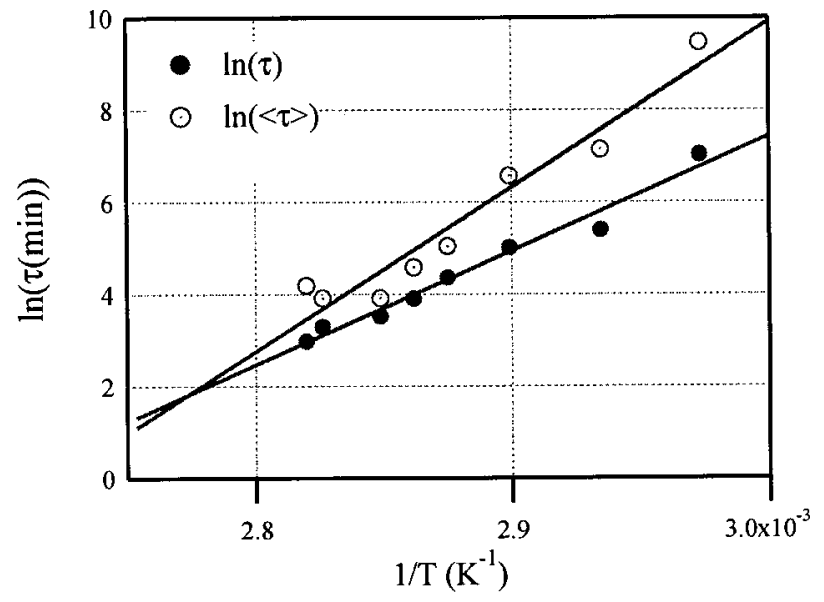

FIG. 2. Natural logarithm of the relaxation time $\tau$ and the average relaxation $\langle\tau\rangle$ obtained with the KWW distribution as a function of $1 /$ temperature. The activation energies obtained are $E_{a}=49 \mathrm{kcal} \mathrm{mol}^{-1}$ for $\tau$, and $E_{a}$ $=71 \mathrm{kcal} \mathrm{mol}^{-1}$ for $\langle\tau\rangle$.

lated on the $\tau$ dependence on $T$ is only $49 \mathrm{kcal} \mathrm{mol}^{-1}$, the difference being due to the fact that the dispersion parameter $\beta$ also varies with $T$ and is taken into account in $\langle\tau\rangle$. An extrapolation to room temperature of the Arrhenius plots leads to the following KWW parameters: $\tau=87 \mathrm{yr}$ and $\langle\tau\rangle$ $=95000 \mathrm{yr}$, thus corresponding to a $\beta$ value of about 0.15 .

\section{Room temperature stability}

A sample stored at room temperature (about $20 \pm 5^{\circ} \mathrm{C}$ ) has been periodically measured over a period of more than 1 yr [Fig. 3(a)]. The experimental data could be fitted with a stretched exponential as seen in Fig. 3(b). The fit parameters obtained are: $\tau=300 \mathrm{~d} ; \beta=0.1 ; \quad(\langle\tau\rangle=3000000 \mathrm{yr})$. As seen in Fig. 3(a), the SHG intensity falls rapidly, but after about $25 \mathrm{~d}$ reaches a value around $40 \%-50 \%$ of its initial value. The efficiency of the sample remains stable for at least $1 \mathrm{yr}$ and, according to the KWW fit, the SHG intensity does not fall an extra $50 \%$ for the next $10 \mathrm{yr}$. Also represented is

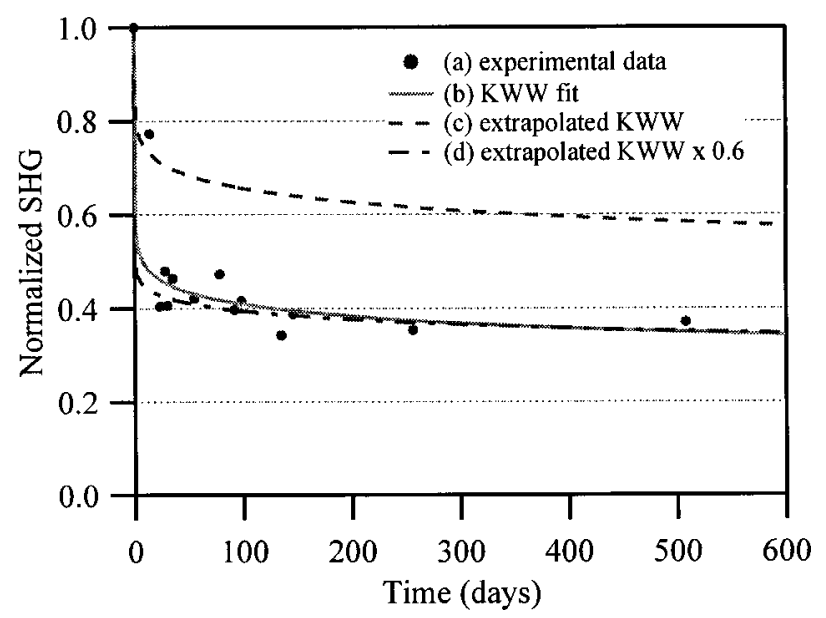

FIG. 3. Logarithm of SHG signal periodically gathered at room temperature over more than 1 yr: (a) experimental data; (b) KWW fit: $\tau=30 \mathrm{~d} ; \beta$ $=0.1$; (c) Arrhenius extrapolated KWW curve: $\tau=87 \mathrm{yr} ;\langle\tau\rangle=95000 \mathrm{yr}$ so, $\beta \approx 0.15$, and (d) same curve multiplied by a factor of 0.6 . 
a stretched exponential whose parameters are previously extrapolated from the Arrhenius plots [Fig. 3(c)]. This last curve passes well above the experimental data. Yet, a factor of 0.6 applied to it enables it to fit the room temperature data, except for the first few points [Fig. 3(d)]. This can be easily explained with the normalization system used for our data. High temperature deorientation was always made with samples polarized about 1 month before, and the normalization value used was the one taken at the beginning of the deorientation cycle. The samples have then always suffered a nonlinear intensity loss of about $40 \%-50 \%$ at room temperature before $t=0$, which is evidently not the case of the sample used for the room temperature deorientation. The extrapolated fit from the Arrhenius plot is indeed in much better agreement with the experimental data shifted at $(t-30$ d.)

Second-order nonlinearity in the hybrid material examined here has a reasonable estimated lifetime at room temperature, which is sufficient for telecom applications. Such application-dependent lifetime, however, has to be meticulously defined and examined. ${ }^{21}$ Also, progress still has to be made for stability at higher temperatures up to $60^{\circ} \mathrm{C}$, but the advantage of the sol-gel technique is that the poling temperature of our material currently under study is still quite low $\left(T_{p}=90^{\circ} \mathrm{C}\right)$. A lifetime of about $12 \mathrm{~d}\left(10^{6} \mathrm{~s}\right)$ at room temperature, for an organic polymer thin film with a glass transition temperature $T_{g}$ of around $80^{\circ} \mathrm{C}$ has been previously reported. ${ }^{2}$ This is similar to another poled amorphous polymer with main-chain chromophores of nominal $T_{g}$ around $80^{\circ} \mathrm{C}$, whose second-order nonlinear stability has been reported to be about $32 \mathrm{~d}(770 \mathrm{~h}){ }^{3}$ It would seem that a hybrid sol-gel material, possessing an inorganic network matrix, would potentially confer greater orientational stability than all-organic materials for an equivalent $T_{g}$.

\section{CONCLUSION}

Reorientational processes of chromophores bound to hybrid inorganic/organic sol-gel matrices are very similar to the ones of chromophores in organic polymers, and the KWW model, using the stretched exponential function, gives an accurate fit of the experimental data. The temperature dependence is found to follow an Arrhenius law. A quick procedure to determine the lifetime of the anisotropic state of a polymer or sol-gel thin film can be suggested: several quick measurements (about a week's time maximum for each) of the SHG decay signal at different temperatures must be made. An analysis by a stretched exponential of the gathered data gives an evaluation of the second-order nonlinearity lifetime for lower temperatures down to room temperature.

The second-order nonlinearity of DR1/SiK/TEOS 5/1/1 sol-gel thin film has been found stable for roughly a decade at room temperature. This bodes well for the applications of these materials in optoelectronics in years to come, especially as these sol-gel materials have not yet been optimized in terms of thermal stability.

${ }^{1}$ Y. Shi, C. Zhang, H. Zhang, J. H. Bechtel, L. R. Dalton, B. H. Robinson, and W. H. Steier, Science 288, 119 (2000).

${ }^{2}$ I. Teraoka, D. Jungbauer, B. Reck, D. Y. Yoon, R. Twieg, and C. G. Willson, J. Appl. Phys. 69, 2568 (1991).

${ }^{3}$ K. D. Singer, in Organic Thin Films for Waveguiding Nonlinear Optics, Advances in Nonlinear Optics Series, edited by F. Kajzar and J. D. Swalen (Gordon and Breach, New York, 1996), Vol. 3, pp. 193-220.

${ }^{4}$ K. Izawa, N. Okamoto, and O. Sugihara, Jpn. J. Appl. Phys., Part 132 , 807 (1993).

${ }^{5}$ H. Hayashi, H. Nakayama, O. Sugihara, and N. Okamoto, Opt. Lett. 20, 2264 (1995).

${ }^{6}$ D. H. Choi, J. H. Park, T. H. Rhee, N. Kim, and S. D. Lee, Chem. Mater. 10, 705 (1998).

${ }^{7}$ F. Chaput, D. Riehl, Y. Lévy, and J. P. Boilot, Chem. Mater. 5, 589 (1993).

${ }^{8}$ L. Ventelon et al., Proc. SPIE 3623, 184 (1999).

${ }^{9}$ G. Williams and D. C. Watts, Trans. Faraday Soc. 66, 80 (1970).

${ }^{10}$ H. Scher, M. Schlesinger, and J. Bendler, Phys. Today 44 (1), 26 (1991).

${ }^{11}$ K. D. Singer and L. A. King, J. Appl. Phys. 70, 3251 (1991).

${ }^{12}$ C. Angell, K. Ngai, G. McKenna, P. McMillan, and W. Martin, J. Appl. Phys. 88, 3113 (2000).

${ }^{13}$ Y. Shuto and M. Amano, J. Appl. Phys. 79, 4358 (1996).

${ }^{14}$ S. H. Lee, J. W. Lee, O. P. Kwon, C. H. Lee, and Y. H. Won, Appl. Phys. Lett. 74, 2067 (1999).

${ }^{15}$ F. Michelotti, E. Toussaere, R. Levenson, J. Liang, and J. Zyss, J. Appl. Phys. 80, 1773 (1996).

${ }^{16}$ R. Dureiko, D. Schuele, and K. D. Singer, J. Opt. Soc. Am. B 15, 338 (1998).

${ }^{17}$ K. Wong and Q. Shen, J. Appl. Phys. 86, 2953 (1999).

${ }^{18}$ A. Dhinojwala, G. K. Wong, and J. M. Torkelson, J. Chem. Phys. 100, 6046 (1994).

${ }^{19}$ F. Michelotti, G. Nicolao, F. Tesi, and M. Bertolotti, Chem. Phys. 245, 311 (1999).

${ }^{20}$ M. Stähelin, C. Walsch, D. Burland, R. Miller, R. Twieg, and W. Volksen, J. Appl. Phys. 73, 8471 (1993).

${ }^{21}$ H. Goudket, M. Canva, Y. Lévy, F. Chaput, and J-P. Boilot, Proc. SPIE 4461-21 (to be published).

${ }^{22}$ D. Imaizumi, T. Hayakama, T. Kasuga, and M. Nogami, J. Sol-Gel Sci. Technol. 19, 383 (2000). 